\title{
Preoperative delirium in patients with fragility fractures: Preliminary results of the detect (delirium in elderly patients admitted to trauma) study protocol
}

\author{
Carlo Rostagno $^{1 *}$, Roberto Civinini' ${ }^{2}$, Maria Ramona Melis ${ }^{1}$, Gianluca Polidori ${ }^{1}$, Claudia Ranalli ${ }^{1}$, Maria Chiara Cavallini ${ }^{3}$, Andrea Ungar ${ }^{3}$ \\ and Alessandro Cartei ${ }^{1}$ \\ ${ }^{1}$ Division of Internal and Post-Surgical Medicine, Azienda Ospedaliero-Universitaria Careggi and University of Florence, Florence, Italy \\ ${ }^{2}$ Division of Traumatology and Orthopedic Surgery, Azienda Ospedaliero-Universitaria Careggi and University of Florence, Florence, Italy \\ ${ }^{3}$ Division of Geriatric Medicine and Cardiology, Azienda Ospedaliero-Universitaria Careggi and University of Florence, Italy
}

\begin{abstract}
Objectives: Creation of a common background that may allow different health care professionals to effectively share clinical information and, at the same time, measuring the relevance of delirium in daily practice.

Methods: We proposed a perspective observational study, with the involvement of all the professionals participating to the process of care, i.e. physicians, nurses, and physiotherapists. Patients were evaluated on admission and at 24 and 48 hours with the Confusion Assessment Method (CAM) and the 4AT for detection of Delirium. Risk factors, predisposing conditions, functional status, and cognitive function are also taken into account, in this paper we report the incidence and factors related to preoperative delirium in trauma elderly patients admitted to our department.

Results: 115 out of 478 patients developed an acute episode of confusion before surgery (24,1\%), 70 within the first 24 hours after hospital admission. Hyperkinetic delirium was more frequent; however, the diagnosis of hypokinetic form may be underestimated. Age, cognitive and functional impairment, sensory deficits are related to development of preoperative delirium.
\end{abstract}

Implications: Occurrence of delirium during hospitalization is a frequent complication and can be considered an adverse health care sequel. Furthermore, it is associated to negative outcomes on mortality, morbidity, functional level and cognitive performance, longer hospital stays and increased risk for institutionalization. Health care costs are also increased for those patients that develop delirium in several hospital settings. A prompt and accurate diagnosis with the introduction of all those preventive and non-pharmacological measures may reduce the incidence, severity and/or duration of delirium and improve patient's functional recovery.

\section{Introduction}

The aging of population as well as the increased life expectancy make progressively urgent demands about the adequacy of the level of care provided in a hospital environment to ensure clinical pathways tailored on the needs of each individual patient. On the other hand, the gradual evolution of Health-care systems to care for the acute, with increasing levels of specialization often technologically driven, cannot and must not neglect these aspects of personalization of care protecting the elderly "frail" patient, because of its intrinsic characteristics [1]. In such a context, evaluation of frailty undertakes not only the meaning of prevention and promotion of quality of life, but it takes in account important issues for patient's safety and the economic weight it entails. Delirium (acute confusion state) is a complex syndrome which frequently occurs in the elderly hospitalized population [2]. Development of Delirium is associated with negative outcomes such as a longer hospital stay, a higher rate of complications, cognitive and functional decline, loss of independence, institutionalization, and, ultimately, death [3]. For skilled healthcare professionals, it represents a challenging condition because a prompt and accurate diagnosis with the introduction of all those preventive and non-pharmacological measures may reduce the incidence, severity and/or duration of Delirium and improve functional recovery [4].
One of the crucial points in addressing delirium's detection and assessment during hospitalization is that the clinical team must be trained for this purpose and all team's members must act and interact uniformly by effectively working in a multidisciplinary fashion to achieve collaboration within and between disciplines [5]. Since September 2011, at the Trauma and Orthopedics Centre of the Azienda OspedalieroUniversitaria Careggi in Florence, a multidisciplinary division for a care pathway based on comprehensive preoperative assessment, including a detailed medical history and a careful physical examination with the identification and stabilization of concomitant clinical problems, as well as a functional, nutritional, and cognitive status evaluation, and postsurgical management was created. Different specialist figures, i.e. geriatricians, internal medicine physician, cardiologist's nurses and physiotherapists concur at the clinical activity in order to reduce the time to theatre, postsurgical complications and hospital length of stay.

${ }^{\star}$ Correspondence to: Carlo Rostagno, Division of Internal and Post-Surgical Medicine, Azienda Ospedaliero-Universitaria Careggi Largo Brambilla, 350134 - Florence, Italy, Tel: +39-055-7948063; E-mail: carlo.rostagno@unifi.it

Key words: delirium management, delirium prevention, education, frail elderly, patient's safety

Received: June 08, 2018; Accepted: June 20, 2018; Published: June 25, 2018 

study protocol

Integration of medical information and interaction with the orthopedic surgeon and the anesthesiologists are required in order to create a safer environment for each patient. Therefore, it is important to develop a teamwork approach in regard to Delirium, creating competence able to jointly produce knowledge rather than reproduce information. Nurses are key figures in management of delirium, in the phases of prevention/ treatment related to non-pharmacological treatment. In this paper we report preliminary results. "The DETEcT Study - Delirium in Elderly patients “(ClinicalTrials.gov identifier: NCT02086981).

\section{Design and methods}

A prospective observational study has been designed. All consecutive patients aging $>65$ years without distinction of gender, admitted in the traumatology ward of the Department of Orthopedics and Traumatology of Careggi Hospital in Florence, were considered eligible. Exclusion criteria are: the impossibility to give an informed consent or refusal to participate to the study. Main objectives were to determine preoperative incidence and prevalence of Delirium and its association with risk factors and their prognostic value. On admission demographic characteristics (age, sex, family composition and presence of a care-giver) were collected. Cognitive status is assessed through the Informant Questionnaire on Cognitive Decline in the Elderly (IQCODE) compiled by a care-giver, while functional independence with the Katz Index of Independence in Activities of Daily Living (ADL) and the Lawton Instrumental Activities of Daily Living (IADL) Scale [6-9]. Mobility is evaluated with the Modified Barthel Index for the walking domain. The presence of common chronic disease conditions, including coronary artery disease, congestive heart failure, atrial fibrillation, hypertension, diabetes mellitus and respiratory failure is collected [10]. Number of medications and modifiable and non-modifiable risk factors for delirium are recorded [11]. On hospital admission and every 24 hours for the next two days or in case of a variation of the clinical condition, patients are administered by trained personnel the Confusion Assessment Method (CAM) and the 4 AT Scale for the identification of Delirium [12,13]. Medical data also include ASA score for surgical risk, the type of fracture, time to surgery as well as peri and post-operative complications, including pressure ulcer rate and nosocomial infection, use of blood transfusions and hospital length of stay [14]. Discharge destination was also reported. The study protocol has been approved by the local ethics committee and informed consent was obtained by each patient enrolled.

\section{Statistical analysis}

Values for continuous variables were reported as the mean and standard deviation (SD). Categorical variables were compared using the $\chi^{2}$ test or the Fisher exact test (two-tailed). Continuous variables were compared between the groups using the Student $t$ test (twotailed). The relation between continuous variables was examined using Pearson's linear method Statistical analysis was performed using SPSS 18.0 (Chicago, IL, USA) statistical software. A probability value of $<$ 0.05 was statistically significant.

\section{Results}

Between April 31st and July 31st, 2014553 patients were admitted to the Department of Orthopedics and Traumatology of Careggi Hospital in Florence: According to inclusion criteria reported in method section 478 were enrolled in the study. Female gender was significantly more represented (346 vs 142). One third of patients were aged $>85$ years (Table 1). 115 patients developed an acute episode of confusion before surgery $(24,1 \%), 70$ within the first 24 hours after hospital admission.
Hyperkinetic delirium was more frequent; however, the diagnosis of hypokinetic form may be underestimated (Table 2).

Development of delirium was significantly more frequent in patients with hip fracture $(66.1 \%)$ in comparison to other sites of fracture (Tables 3 and 4). Incidence of delirium increased with the age, being less than $1 \%$ in patients $<70$ years and more than $30 \%$ in those aged $>85$ years $(\mathrm{p}<0.0001)$. Cognitive and functional impairment, previous falls and multiple pharmacotherapy were significantly more frequent in patients with delirium (Table 5). The relation with modifiable risk factors and development of delirium is reported in Table 6. Hearing loss and impaired vision were significantly related to the occurrence of delirium. An history of dementia, previous episodes of delirium, gait disturbances, an history of falls and finally Parkinson disease were also related with preoperative delirium.

Table 1. Age distribution of enrolled patients

\begin{tabular}{|c|c|c|}
\hline Mean age & $81.4 \pm 8.4$ years & \\
\hline Age classes (years) & $\mathbf{N}$ & $\%$ \\
\hline $\mathbf{6 5 - 6 9}$ & $\mathbf{5 3}$ & $\mathbf{1 1 . 1}$ \\
\hline $\mathbf{7 0 - 7 4}$ & $\mathbf{5 8}$ & $\mathbf{1 2 . 1}$ \\
\hline $75-79$ & $\mathbf{8 1}$ & $\mathbf{1 6 . 9}$ \\
\hline $\mathbf{8 0 - 8 4}$ & $\mathbf{8 8}$ & $\mathbf{1 8 . 4}$ \\
\hline $\mathbf{8 5 - 8 9}$ & $\mathbf{1 1 6}$ & $\mathbf{2 4 . 3}$ \\
\hline$>=90$ & $\mathbf{8 2}$ & 17.2 \\
\hline
\end{tabular}

Table 2. Incidence and clinical characteristcs of preoperative delirium

\begin{tabular}{|c|c|}
\hline Delirium & Total \\
\hline & $115(24.1)$ \\
\hline Hiperkintec (\%) & $70(60.9)$ \\
\hline Mixed (\%) & $30(26.1)$ \\
\hline Hypokinetic (\%) & $15(13.0)$ \\
\hline
\end{tabular}

Table 3. Site of fracture

\begin{tabular}{|c|c|c|}
\hline & N & $\%$ \\
\hline Upper limb & 83 & 17.4 \\
\hline Lower limb & 47 & 9.8 \\
\hline Column & 19 & 4.0 \\
\hline Hip fracture & 275 & 57.5 \\
\hline Pelvis & 12 & 2.5 \\
\hline Head trauma & 19 & 4.0 \\
\hline Other & 23 & 4.8 \\
\hline Total+ & 478 & 100.0 \\
\hline
\end{tabular}

Table 4. Relation between incidence of delirium and site of fracture

\begin{tabular}{|c|c|c|c|c|c|c|c|c|}
\hline & $\begin{array}{c}\text { Upper } \\
\text { limb }\end{array}$ & $\begin{array}{c}\text { Lower } \\
\text { limb }\end{array}$ & Column & $\begin{array}{c}\text { Hip } \\
\text { fracture }\end{array}$ & Pelvis & $\begin{array}{c}\text { Head } \\
\text { trauma }\end{array}$ & Other & Total \\
\hline No (\%) & $67(18.5)$ & $38(10.5)$ & $17(4.7)$ & $\begin{array}{c}199 \\
(54.8)\end{array}$ & $7(1.9)$ & $15(4.1)$ & $20(5.5)$ & 363 \\
\hline Yes (\%) & $16(13,9)$ & $9(7.8)$ & $2(1.7)$ & $76(66.1)$ & $5(4.3)$ & $4(3.5)$ & $3(2.6)$ & 115 \\
\hline Total & 83 & 47 & 19 & 275 & 12 & 19 & 23 & 478 \\
\hline
\end{tabular}

Table 5. Relation between preoperative delirum and mobilty, history of falls, ADL and IADL and repectively number of drugs

\begin{tabular}{|c|c|c|c|c|}
\hline & \multicolumn{2}{|c|}{ Delirium no } & Delirium yes & p \\
\hline $\begin{array}{c}\text { Functional capacity (Barthel } \\
>/ \leq 2)\end{array}$ & \multicolumn{2}{|c|}{$70 / 293(19.3)$} & $60 / 55(52.2)$ & .000 \\
\hline History of falls $>/ \leq \mathbf{2})$ & $20 / 343$ & $(5.5)$ & $20 / 95(17.4)$ & .000 \\
\hline ADL $(\leq />\mathbf{3})$ & $54 / 309(14.9)$ & $69 / 46(60.0)$ & .000 \\
\hline IADL $(\leq />\mathbf{4})$ & $109 / 254$ & $(30.0)$ & $80 / 35(69.6)$ & .001 \\
\hline Number of drugs $(>/ \leq \mathbf{3})$ & $158 / 205$ & $(43.5)$ & $68 / 47(59.1)$ & .003 \\
\hline
\end{tabular}

$\$ 451 \mathrm{pz}$ 
Table 6. Relation between preoperative delirum and modifiable factors

\begin{tabular}{|c|c|c|c|c|}
\hline Modifiable & & Delirium no & Delirium yes & P \\
\hline \multirow{2}{*}{$\begin{array}{c}\text { Sensorial } \\
\text { impairment }\end{array}$} & Hearing loss & $94 / 269(25.9)$ & $66 / 49(57.4)$ & 0 \\
\cline { 2 - 5 } & $\begin{array}{c}\text { Visual } \\
\text { impairment }\end{array}$ & $61 / 302(16.8)$ & $41 / 74(35.7)$ & 0 \\
\cline { 2 - 5 } & Malnutrition & $48 / 315(13.2)$ & $31 / 84(24.0)$ & 0.001 \\
\hline Not modifiable & & Delirium no & Delirium yes & p \\
\hline Dementia & & $23 / 340(6.3)$ & $48 / 67(41.7)$ & 0.001 \\
\hline \multirow{2}{*}{ History of } & Delirium & $6 / 357(1.7)$ & $19 / 96(16.5)$ & 0 \\
\cline { 2 - 5 } & $\begin{array}{c}\text { Galls } \\
\text { abnormait }\end{array}$ & $55 / 308(15.2)$ & $34 / 81(29.6)$ & 0.001 \\
\hline \multirow{2}{*}{ Comorbidity } & $\begin{array}{c}\text { Parkinson } \\
\text { disease }\end{array}$ & $57 / 306(15.7)$ & $37 / 78(32.2)$ & 0 \\
\hline
\end{tabular}

\section{Discussion}

Occurrence of delirium during hospitalization leads to both poor clinical and functional outcomes and frequent adverse events. There is also growing evidence that health care costs are increased for those patients that develop delirium in several hospital settings. In a prospective cohort study in 275 consecutive mechanically ventilated patients was found a 39\% increase of intensive care unit costs (95 confidence interval, (CI) $12-72 \%$ ) and $31 \%$ greater hospital costs (95\% CI, 1-70\%) if compared to a non-delirious control group, after adjusting for confounding variables including age, co-morbidities and severity of illness, degree of organ dysfunction, nosocomial infection, and hospital mortality [15]. A 1-year health care costs observation associated with delirium in an elderly population showed that total costs per day survived were more than 2.5 times higher for patients with delirium than for patients without delirium [16]. The overall financial impact of delirium on the health care system ranged from $\$ 38$ to $\$ 152$ billion annually. It is therefore evident that the economic burden of delirium is substantial, and it is a serious condition with longterm clinical and economic implications. In elderly patient's candidate for surgery a substantial number of patients develops delirium before surgery and this may lead to surgery delay and higher post-operative complication rate. Preliminary data from our survey suggest that one fourth of patients aged $>645$ years develop delirium before surgery, most within 24 years from admission. Although not modifiable factors (age, comorbidities, functional impairment) are significantly related to the development of preoperative delirium, however it must be stressed that impaired vision and hearing are also significantly related to behavioral abnormalities. Help to these patients by careful nursing may prevent the soccurrence of delirium. These data support the results from Inouye, et al [12]. Finally, as indicated by the Institute of Medicine, drawing upon data gathered directly from the patient's care environment has enormous potential for accelerating the rate at which useful knowledge is produced, including information about quality of care, efficiency, safety, and cost-effectiveness [17]. Patient's outcome can be improved by putting into practice preventive strategies and particularly multicomponent approaches and optimization of risk factors lead to reduced incidence. Appropriate treatment of delirium requires thorough investigation, management of the underlying illness, avoidance of complications and simplification of the care environment.

\section{Declarations}

ClinicalTrials.gov identifier: NCT02086981. “The DETEcT Study Delirium in Elderly paTiEnts Admitted to Trauma” - http://clinicaltrials. gov/ct2/show/NCT02086981

\section{References}

1. Morley JE, Haren MT, Rolland Y, Kim MJ (2006) Frailty. Med Clin North Am 90 837-847. [Crossref]

2. Flaherty JH (2011) The evaluation and management of delirium among older persons. Med Clin North Am 95: 555-577. [Crossref]

3. Inouye SK, Westendorp RG, Saczynski JS (2014) Delirium in elderly people. Lancet 383: 911-922. [Crossref]

4. Young J, Leentjens AF, George J, Olofsson B, Gustafson Y (2008) Systematic approaches to the prevention and management of patients with delirium. $J$ Psychosom Res 65: 267-272. [Crossref]

5. Ramaswamy R, Dix EF, Drew JE, Diamond JJ, Inouye SK, et al. (2011) Beyond grand rounds: a comprehensive and sequential intervention to improve identification of delirium. Gerontologist 51: 122-131. [Crossref]

6. Jorm AF (1994) A short form of the Informant Questionnaire on Cognitive Decline in the Elderly (IQCODE): development and cross-validation. Psychol Med 24:145-153. [Crossref]

7. Cherbuin N, Anstey KJ, Lipnicki DM (2008) Screening for dementia: a review of selfand informant-assessment instruments. Int Psychogeriatr 20: 431-458. [Crossref]

8. Katz S, Ford AB, Moskowitz RW, Jackson BA, Jaffe MW (1963) Studies of illness in the aged. the index of adl: A standardized measure of biological and psychosocial function. JAMA 185: 914-919. [Crossref]

9. Lawton MP, Brody EM (1969) Assessment of older people: self-maintaining and instrumental activities of daily living. Gerontologist 9: 179-186. [Crossref]

10. Shah S, Vanclay F, Cooper B (1989) Improving the sensitivity of the Barthel Index for stroke rehabilitation. J Clin Epidemiol 42: 703-709. [Crossref]

11. Fong TG, Tulebaev SR, Inouye SK (2009) Delirium in elderly adults: diagnosis, prevention and treatment. Nat Rev Neurol 5: 210-220. [Crossref]

12. Inouye SK, van Dyck CH, Alessi CA, Balkin S, Siegal AP, et al. (1990) Clarifying confusion: the confusion assessment method. A new method for detection of delirium. Ann Intern Med 113: 941-948. [Crossref]

13. Bellelli G, Morandi A, Davis DH, Mazzola P, Turco R, et al. (2014) Validation of the 4AT, a new instrument for rapid delirium screening: a study in 234 hospitalised older people. Age Ageing 43: 496-502. [Crossref]

14. Saklad M (1941) Grading of patients for surgical procedures. Anesthesiol 2:281-284.

15. Milbrandt EB, Deppen S, Harrison PL, Shintani AK, Speroff T, et al. (2004) Costs associated with delirium in mechanically ventilated patients. Crit Care Med 32: 955962. [Crossref]

16. Leslie DL, Marcantonio ER, Zhang Y, Leo-Summers L, Inouye SK (2008) One-year health care costs associated with delirium in the elderly population. Arch Intern Med 168: 27-32. [Crossref]

17. Grossman C, Goolsby AW, Olsen L, McGinnis M (2010) Clinical Data as the Basic Staple of Health Learning: Creating and Protecting a Public Good. National Academies Press Washington, DC USA.

Copyright: (C2018 Rostagno C. This is an open-access article distributed under the terms of the Creative Commons Attribution License, which permits unrestricted use, distribution, and reproduction in any medium, provided the original author and source are credited. 